\title{
La Medicina Interna en España en la crisis del COVID-19
}

\author{
Internal Medicine during the COVID-19 crisis in Spain
}

\author{
Carlos Mª San Román Terán ${ }^{1}$, Ricardo Gómez-Huelgas² \\ ${ }^{1}$ Servicio de Medicina Interna. Hospital General Básico de la Axarquía. ${ }^{2}$ Servicio de Medicina Interna. Hospital Regional Universitario de Málaga.
}

\section{La Medicina Interna en España en la era pre-COVID-19. ¿De dónde partíamos?}

Las unidades y servicios de Medicina Interna españoles atienden toda una amplia variedad de patologías médicas del adulto, si bien el mayor volumen de pacientes atendidos en régimen de hospitalización lo constituyen los mayores de 65 años y los pacientes portadores de pluripatología o multimorbilidad. En el período 2016-2019 se documentaron en el Registro de Atención Especializada del Ministerio de Sanidad (https://pestadistico.inteligenciadegestion.mscbs.es/publicoSNS/S/rae-cmbd) un total de 17.813.520 episodios de ingreso hospitalario en mayores de 65 años, de los cuales los servicios de especialidades médicas atendieron casi siete millones de casos (6.908.363), que corresponden al 38,8\%, siendo atendidos en Medicina Interna 2.872.585, es decir el $41,6 \%$ de todos los episodios médicos y un $16,1 \%$ del total. El estudio RECALMIN II ${ }^{1}$ aproxima aún más las características de los pacientes atendidos por Medicina Interna, definiendo una edad media de 74 $\pm 16,2$ años y un Índice de Charlson de $4,7 \pm 3,9$ (en datos de 2014), lo que confirma la concentración de pacientes mayores y pluripatológicos en las unidades de Medicina Interna.

Por otro lado, no se le escapa a quien conozca la historia reciente de la actividad asistencial en España, el papel decisivo que las unidades de Medicina Interna desarrollaron en la década de los 80 del pasado siglo durante otras crisis sanitarias como la pandemia $\mathrm{VIH} / \mathrm{SIDA}$ o la intoxicación masiva por aceite de colza desnaturalizado. Igualmente, los servicios de Medicina Interna están habituados a ejercer tareas de armonización de la demanda hospitalaria en las epidemias anuales de gripe.

\section{Papel de la Medicina Interna}

durante la crisis de la COVID-19

Desde los primeros momentos de la pandemia, los servicios de Medicina Interna adquirieron un protagonismo evidente en la atención de los pacientes con infección grave por SARS-CoV-2, y la rápida adaptación de los internistas ante un desafío inédito resultó decisiva para evitar el colapso del sistema hospitalario. La COVID-19 provocó la llegada masiva de pacientes en situación clínica comprometida que requerían ingreso hospitalario debido a una neumonía con enfermedad multisistémica ${ }^{2}$ cuya gravedad sobrepasaba con cierta frecuencia las actuaciones habituales de las unidades convencionales de hospitalización para requerir cuidados especiales con respiración asistida propios de las unidades de Cuidados Intensivos que, a su vez, se vieron sobrepasadas en su capacidad de contención y respuesta, alcanzándose picos de ocupación mantenida durante varias semanas cercana al $80 \%$ del total hospitalario, tanto en hospitales terciarios grandes como de primer nivel. ${ }^{3}$

La versatilidad de los internistas facilitó la rápida expansión de plantas COVID en espacios del hospital asignados a otros servicios, generalmente quirúrgicos, que tuvieron que suspender paulatinamente sus actividades programadas. A medida que se fueron llenado los hospitales de pacientes COVID-19, las plantillas de internistas dejaron de ser suficientes para la atención de tal número de pacientes graves, por lo que otros especialistas médicos y quirúrgicos que vieron mermada su actividad se fueron incorporando a los equipos COVID multidisciplinarios que, liderados generalmente por internistas y apoyados por la enfermería de cada unidad, formaron rápida y ordenadamente grupos de muy alta capacidad en el diagnóstico, cuidado y tratamiento de los pacientes, asumiendo los diversos y sucesivos cambios de indicaciones terapéuticas que la COVID-19 fue generando conforme a los conocimientos y evidencias emergentes. ${ }^{4}$

La rápida reorganización hospitalaria para dar respuesta a la pandemia fue un esfuerzo colectivo liderado por el ejemplar compromiso y el sacrificio de los profesionales sanitarios que asumieron desde el inicio la necesidad de establecer un nuevo modelo asistencial basado en el proceso asistencial, con la configuración de equipos multidisciplinares, el establecimiento de triages, la ordenación en diferentes niveles de cuidados con una adecuada continuidad asistencial, el desarrollo de protocolos asistenciales comunes y dinámicos en base a las evidencias que, a un ritmo vertiginoso, se iban generando de una enfermedad desconocida, y la implementación de la telemonitorización y teleasistencia domiciliarias, tanto de los pacientes COVID de menor gravedad como de aquellas patologías no COVID que requerían una atención no demorable. 


\section{"debe resaltarse el meritorio esfuerzo investigador que han realizado los internistas españoles durante la pandemia, a pesar de la enorme carga asistencial que estaban soportando"}

Pero no es menos cierto que la tradición de aproximación holística integral al paciente de los médicos internistas y su adaptabilidad clínica fueron determinantes para dar un enfoque adecuado a una enfermedad infecciosa con manifestaciones multisistémicas, para dar un enfoque terapéutico multifactorial (antiviral, antiinflamatorio, antitrombótico) y para la utilización sistemática y generalizada de técnicas habitualmente reservadas a especialidades de atención crítica que comenzaron a formar parte del arsenal terapéutico habitual, como la oxigenación de alto flujo, la ventilación mecánica no invasiva y la CPAP.

Asimismo, debe resaltarse el meritorio esfuerzo investigador que han realizado los internistas españoles durante la pandemia, a pesar de la enorme carga asistencial que estaban soportando. El Registro SEMI-COVID-19 es una muestra del compromiso profesional de más de 600 internistas pertenecientes a 150 hospitales españoles. Actualmente se han incluido más 20,000 pacientes hospitalizados por COVID-19 y constituye una de las mayores bases de datos a nivel mundial. El Registro SEMI-COVID-19 está generando una importante producción científica (https://pubmed.ncbi.nlm.nih.gov/collectio ns/60175415/?sort=pubdate) que contribuye a un mejor entendimiento de los aspectos clínicos, diagnósticos, terapéuticos y pronósticos de una patología nueva y desconocida.

Con el análisis global del Registro SEMI-COVID-19², la impresión que se desprende de la actuación de la mayoría de las unidades de Medicina Interna podía acercarse a lo que Seymur llama "Medicina Sensata", que se podría resumir como medicina prudente que permite esperar con paciencia a la evidencia de las actuaciones, pero sin dejar de usar todas las herramientas que ya hayan mostrado su interés, atentos a los efectos secundarios, detallando cada decisión terapéutica y su efectividad y, en general, con una visión más suave y humilde de las opciones disponibles ${ }^{4}$.

\section{Enseñanzas para el futuro}

La pandemia de COVID-19 ha generado un drama colectivo y una catástrofe económica que marcará a toda una generación. Pero, al igual que ha ocurrido con otros momentos críticos en la historia de la humanidad, representa una oportunidad única de mejora. La pandemia va a acelerar importantes avances terapéuticos y debería reorientar el sistema sanitario a un modelo más integrador, personalizado, eficiente y de alto valor para el ciudadano. Entre las enseñanzas de la atención al COVID-19 podemos destacar: 1) La orientación completa de la asistencia al paciente y a los los resultados asistenciales. 2) El protagonismo de los servicios clínicos sobre el tradicional modelo gerencialista. 3) La superación de las barreras entre las especialidades y la puesta en valor de la colaboración multiprofesional. 4) La evaluación integral del paciente, con el empleo sistemático de sistemas de estratificación del riego y de los niveles de cuidados. 5) El establecimiento de flujos dinámicos entre niveles asistenciales (urgencias-hospitalización-cuidados críticosseguimiento domiciliario). 5) La generalización de la telemedicina. 6) La innovación tecnológica. 7) La desburocratización de la asistencia y de las prestaciones farmacéuticas, y 8) La existencia de una inaceptable fragilidad de los centros sociosanitarios. Muchos de estos cambios hacia un hospital «fluido» o «flexible» con adaptación continua, marcarán el hospital del próximo futuro 5 .

Como reflexión final, la pandemia nos ha permitido realizar una renovación colectiva de los compromisos éticos de la profesión, otorgándonos el privilegio de sentirnos orgullosos de ser médicos, y orgullosos de ser internistas.

\section{BIBLIOGRAFÍA}

1. Zapatero-Gaviria A, Barba-Martín R, Canora Lebrato J, Fernández-Pérez C, Gómez-Huelgas R, Bernal-Sobrino JL,. RECALMIN II. Eight years of hospitalisation in Internal Medicine Units (2007-2014). What has changed? Rev Clin Esp. 2017; 217(8): 446-453.

2. Wiersinga WJ, Rhodes A, Cheng AC, Peacock SJ, Prescott HC. Pathophysiology, Transmission, Diagnosis, and Treatment of Coronavirus Disease 2019 (COVID-19): A Review. JAMA. 2020; 324(8): 782-793.

3. Casas-Rojo JM, Antón-Santos JM, Millán-Núñez-Cortés J, Lumbreras-Bermejo C, Ramos-Rincón JM, Roy-Vallejo E et al; en nombre del Grupo SEMI-COVID-19 Network. Clinical characteristics of patients hospitalized with COVID-19 in Spain: Results from the SEMI-COVID-19 Registry. Rev Clin Esp. 2020; 220(8): 480-494.

4. Seymour CW, McCreary EK, Stegenga J. Sensible Medicine-Balancing Intervention and Inaction During the COVID-19 Pandemic. JAMA. 2020 Oct 15. (Epub ahead of print) doi: 10.1001/jama.2020.20271.

5. García-Alegría J, Gómez-Huelgas R. COVID-19 disease: the hospital of the future is already here. Rev Clin Esp. 2020 0ct;220(7):439-441. 